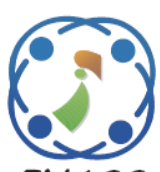

\title{
Weber's Law based Camera Response Model for Low Light Image Contrast Enhancement
}

\author{
Gutta Srinivas Rao ${ }^{1 *}$ \\ Atluri Srikrishna ${ }^{2}$ \\ Nagesh Vadaparthi ${ }^{3}$ \\ ${ }^{1,2}$ Department of Information Technology, R.V.R. \& J.C. College of Engineering, India \\ ${ }^{3}$ Department of Information Technology, \\ Maharaj Vijayaram Gajapathi Raj (MVGR) College of Engineering (Autonomous), India \\ * Corresponding author's Email: gutta.gsr@gmail.com
}

\begin{abstract}
In the field of digital image processing, Image Contrast Enhancement (ICE) is an emerging research area, which helps to attain the sub-standard quality images. The existing ICE techniques have a few limitations like brightness preservation and contrast limitation. Also, the existing ICE techniques affects from over-enhancement that leads to unrealistic results and halo-like artifacts in the high contrast region. To address these issues, a new contrast enhancement method (Second Order Derivative with Weber's Law (SODWL)) is developed in this research study. The proposed SODWL method includes two major phases (i) estimate the relationship between different image exposures and identify the appropriate Camera Response Model (CRM), (ii) illumination estimation method is applied to calculate the exposure ratio map. The SODWL method improved the performance of ICE effectively, because the developed approach considers even the small edge pixel values for contrast enhancement. After that, Weber's law is applied for adjusting the lighting conditions of the input image. The experimental analysis was conducted on the reputed datasets: VV, LIME-data, DICM, and MEF. Finally, the performance of proposed method is evaluated by applying the evaluation metrics such as, Lightness Order Error (LOE), and Visual Information Fidelity (VIF). The experimental result showed that the proposed system improved the VIF value upto 0.4-0.1 and also reduced the LOE value up to 2447-2 as related to the existing systems.
\end{abstract}

Keywords: Camera response model, Contrast, Illumination, Lightness order error, Weber's law.

\section{Introduction}

ICE is an important image processing technique for both human perception and computer vision. It is extensively applicable in the different domain for example, video surveillance system, medical image processing [1], study of satellite images [2], exclusion of haze in transportation system [3, 4], and so on. The significant responsibility of ICE is to increase the contrast level in images for achieving the sub-standard quality images. However, there are several factors reducing the image quality like illumination, contrast and noisy data in image collection phase [5]. The low-contrast effect reduces the visual quality of an image. Hence, ICE is used to recognize the edges of image objects accurately. It is essential to optimize the contrast of an image in order to preserve all the information present in the image. Maximizing the contrast delivers better observation in the images, which is known as ICE. Also, it efficiently enlarges the dynamic range of vital objects in an image. Moreover, ICE methods effectively enhance the image quality with efficient features in computer vision systems [6]. In image processing, ICE method is the primary phase to enhance the image quality efficiently in both human and system level observations. Numerous researchers used several existing methods for ICE; Histogram Equalization [7], Fuzzy system [8, 9], Wavelet Transform [9, 10], etc.

In previous researches, many traditional ICE methodologies are used for achieving the substandard quality images. But, the existing ICE strategies have several limitations, for example, the existing histogram equalization method maximizes 
the contrast of background noise, while removing an unusable input. Some of the other limitations are over the illumination issue [11], low lighting conditions, poor quality, noisy input data and etc. which degrade the performance of ICE [12]. In this paper, an efficient CRM model is proposed, namely SODWL for enhancing the ICE. The proposed framework includes several processes such as CRM, Map Estimation, and Lightening Distortion. At first, CRM allows to adjust the exposure of the input image. Next, estimate the exposure ratio map with the help of illumination estimation techniques. Finally, the Map estimation process helps to map the similar pixel weights and Weber's law is used for adjusting the lighting conditions in an input image. The proposed method helps to calculate the small as well as the large size of pixel illumination weights to improve the ICE.

This paper is composed as follows. Section II presents a survey of several recent papers on ICE. In section III, an effective contrast enhancement method: CRM with Weber's law is described. Section IV shows the comparative experimental result for proposed and existing image enhancement strategies using HDR dataset. The conclusion is made in section $\mathrm{V}$.

\section{Related work}

Several researchers suggested many techniques for image contrast enhancement. A brief evaluation of some essential contributions to the existing literatures is presented in this section.

X. Liu, G. Cheung, X. Ji, D. Zhao, and W. Gao, [13] presented an efficient image enhancement methodology; graph Retinex for decomposing the illumination and reflectance components from the target image. Then, every component was defined mathematically and represented in the graph. In this paper, Accelerated Proximal Gradient (APG) method was used for estimating the illumination and reflectance patch to improve the tracking speed of patches. Next, the graphs were constructed with the help of a number of pixel patches specifically, positive edges were linked to the same pixels for reducing the noise. Similarly, negative edges were linked to the dissimilar pixels for empathizing the image enhancement. In this research study, the developed methodology manually calculates the positive and negative edge weights, so it consumes more computational time.

S. Guo, T. Xiang, and X. Li, [14] presented a Multi-Scale Fuzzy Gradient Similarity Deviation (MFGSD) method to estimate the image quality. The advanced MFGSD methodology used the fuzzy interference system for decreasing the destructive influences of invisible noise. In addition, the developed system calculates the image quality differences with the help of fuzzy gradient similarity metric. The experimental analysis was conducted on different reputed datasets. Also, the computational cost and errors was measured in a multi-scale image. While using multiple datasets, the MFGSD score gets maximized that highly affects the image quality.

C. Jung, Q. Yang, T. Sun, Q. Fu, and H. Song, [15] presented an efficient ICE approach namely Dual-Tree Complex Wavelet Transform (DT-CWT), which was used in many ranges of images without noise extension. For image enhancement, Contrast Limited Adaptive Histogram Equalization (CLAHE) method was used in this research study. According to the experimental outcome, the DT-CWT method clearly indicates the different natures of input images such as informative, visually pleasing, and informative on a low light image. Also, the developed method significantly improved the image quality and also effectively reduced the noise. The developed DT-CWT approach effectively decreases the noise in luminance channel, but showed limited performance in chrominance channel.

S. Zhang, T. Wang, J. Dong, and H. Yu, [16] developed an efficient approach: LAB-Multi-Scale Retinex (LAB-MSR) for image enhancement. The LAB-MSR method used hybrid bilateral filter and trilateral filter in the CIELAB color space in order to arrange every channel based on their characteristics. The LAB-MSR method resolve the image brightness issues and also effectively eliminated high contrast from the image. In the case of halo artifacts, the developed approach averagely improved the image quality, still it needs to be concentrated.

J. Cai, S. Gu, and L. Zhang, [17] developed Convolutional Neural Network (CNN) method to train the Single ICE (SICE). The major problem in ICE is to construct the low and high contrast image in the learning process of the training set. In order to rectify the problem, the $\mathrm{CNN}$ based learning process was used. Moreover, CNN based SICE method effectively achieve the maximum quality of improved results in an underexposed input image. However, the developed method failed to recover the details for large and severely overexposed regions.

Z. Ying, G. Li, and W. Gao, [18] designed a weight matrix for image fusion by utilizing an illumination estimation approach. In addition, camera response model was applied for synthesizing the multi-exposure images. Then, identify the best exposure ratio, so the synthetic images were well exposed in the regions where the original images 
were underexposed. At last, the enhanced result was attained by combining the synthetic image and the input image on the basis of weight matrix. The experimental outcome showed that the developed approach gives better result related to the existing systems. The developed system involves human intervention, which was considered as one of the major drawbacks.

Y. Ren, Z. Ying, T.H. Li, and G. Li, [19] developed a new enhancement system by using the response features of cameras. At first, determine the reasonable camera response model and its features. Then, illumination estimation methods were utilized to identify the exposure ratio for each pixel. At last, the selected camera response model was utilized for adjusting each pixel to the desired exposure based on the estimated exposure ratio map. Experimental outcome shows that the developed method obtains enhancement results with fewer color and lightness distortions related to other existing systems. The developed system considered only a few camera features, which need to be increased for further improving the performance of ICE.

An efficient ICE technique (CRM-Weber's Law) is implemented in this paper to overcome the above-mentioned drawbacks and for enhancing the performance of HDR image contrast enhancement.

\section{Proposed methodology}

The proposed SODWL based ICE technique includes two major tasks; identification of appropriate CRM and determine the exposure ratio map. The SODWL based ICE technique identifies the histogram features of the two different images that vary in exposure. Next, calculation of illumination methods measures the exposure ratio map in the input image. Finally, the proposed image enhancement algorithm performed based on the CRM and the estimated exposure ratio map. The proposed architecture is shown in Fig. 1.

\subsection{Camera response model}

Generally, camera manufacturers are using some nonlinear features in camera, for example, white balance and demosaicing to enhance the visual quality of the captures images. The CRM consists of two major components Brightness Transform Function (BTF) and Camera Response Function (CRF). The parameters of CRF model are determined only by camera while BTF model is determined by the camera and exposure ratio. Initially BTF model performed based on the observation of two different exposure images. Then

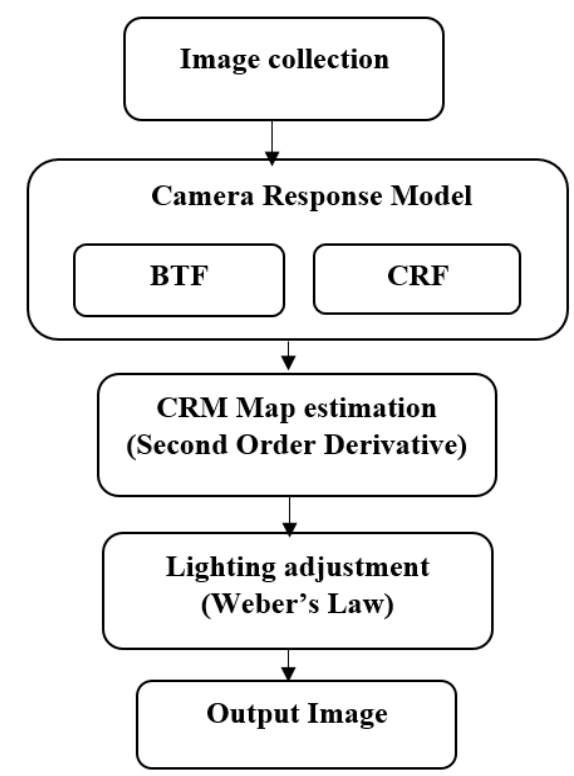

Figure.1 Proposed architecture

derive the corresponding CRF model by solving the comparametric equation. These two functions are described in the following section.

\subsubsection{Brightness transform function (BTF)}

At first, BTF selects two images such as $P_{0}$ and $P_{1}$ to calculate the BTF and its value vary only in exposure. After that, construct the histogram for each color channel in the input image. According to the histogram plot, under-exposed image highly concentrated in low brightness area. The gamma values represent the linear amplification of pixels of the resultant image which is close to the real wellexposed image. However, two parameter functions used to define the BTF model and it's mathematically described in Eq. (1).

$$
P_{1}=g\left(P_{0}, k\right)=\beta P_{0}^{\gamma}
$$

Where $\beta$ and $\gamma$ are parameters in the BTF model related to exposure ratio $k$. The observation also shows that different color channels have same model parameters, because the response curve of various color channels is nearly identical.

\subsubsection{Camera response function (CRF)}

The CRF model calculates the relationship between the BTF model parameters such as $\beta$ and $\gamma$. The CRF model is derived by solving the following comparametric Eq. (2).

$$
f(k E)=\beta f(E)^{\gamma}
$$


The closed-form solution of $f$ is provided in following Eq. (3).

$$
f(E)=\left\{\begin{array}{c}
e^{b\left(1-E^{a}\right)}, \text { if } \gamma \neq 1 \\
E^{c}, \text { if } \gamma=1
\end{array}\right.
$$

Whereas, $a$ and $b$ are model parameters in the case of $\gamma \neq 1$ :

$$
a=\log _{k} \gamma, b=\frac{\ln \beta}{1-\gamma}
$$

In addition, $\mathrm{c}$ is a model parameter in the case of $\gamma=1$, Eq. (5)

$$
c=\log _{k} \beta
$$

Two CRF models can be derived from two cases of Eq. 3. When $\gamma=1$, the CRF model becomes a power function and the BTF model becomes a simple linear function. As some camera manufacturers design $f$ to be a gamma curve, it can fit these cameras perfectly. When $\gamma \neq 1$, the CRF model becomes a two-parameter function and the BTF model becomes a non-linear function.

\subsection{Mapping function}

The $a$ and $b$ parameters of BTF model is measured in the Eq. (4). The BTF model is derived in Eq. (6).

$$
\beta=e^{b\left(1-k^{a}\right)}, \gamma=k^{a}
$$

Since the camera response curve is fixed for a specific camera and the parameters $(a$ and $b)$ are obtained by fitting the curve. In the camera response model, the input image is indicated as $P_{0}$ and arbitrary exposure ratio $k_{*}$, and corresponding image $P_{*}$ that differ only in exposure from BTF model in Eq. (7).

$$
g\left(P_{0}, k_{*}\right)=P_{*}=\beta_{*} P_{0}^{\gamma_{*}}=e^{b\left(1-k_{*}{ }^{a}\right)} P_{0}^{k_{*}{ }^{a}}
$$

There are numerous illumination estimation methods proposed for image enhancement task. Where $\beta$ and $\gamma$ are two model parameters that can be calculated from camera parameters $a, b$ and exposure ratio $k$. Assume that no information about the camera is provided and use a fixed camera parameter $(a=-0: 3293 ; b=1: 1258)$ that can fit most cameras. The lightness component as the initial estimation of illumination is shown in Eq. (8).

$$
L(x)=\max _{c \in\{R, G, B\}} P_{c}(x)
$$

For each individual pixel $x$, calculate the weight matrix as shown in the Eq. (9).

$$
W_{d}(x)-\frac{1}{\left|\sum_{y \in w(x)} \nabla_{d} L(y)\right|+\epsilon}, d \in\{h, v\}
$$

Where $|*|$ is the absolute value operator, $\omega(x)$ is the local window centered at the pixel $x$ and $\epsilon$ is a very small constant to avoid the zero denominator. The first-order derivative filter $\nabla d$ contains $\nabla h$ (horizontal) and $\nabla v$ (vertical). The refined illumination map $T$ is solved by optimization which is shown in Eq. (10).

$$
\begin{gathered}
\min _{T} \sum_{x}\left((T(x)-L(x))^{2}+\right. \\
\left.\lambda \sum_{d \in\{h, v\}} \frac{W_{d}(x)\left(\nabla_{d} T(x)\right)^{2}}{\left|\nabla_{d} L(x)\right|+\epsilon}\right)
\end{gathered}
$$

Where, $\lambda$ is the coefficient to balance the involved terms such as illumination map and pixel values. According to the map estimation, at first calculate the image illumination map which is indicated as $T$. Therefore, the result can be directly computed without any iterations. In first order derivative, small pixel values are eliminated that degrades the performance. In order to detect the small pixel values, second order derivative is used. The second order derivative filter is mathematically shown in Eq. (11).

$$
\begin{array}{r}
\min _{T} \sum_{x}\left((T(x)-L(x))^{2}+\right. \\
\left.\lambda \sum_{d \in\{h, v\}} \frac{W_{d}(x)\left(\nabla_{d}^{2} T(x)\right)^{2}}{\left|\nabla_{d}^{2} L(x)\right|+\epsilon}\right)
\end{array}
$$

Whereas, the second order derivative function calculates the pixel values of horizontal and vertical of the input image. 


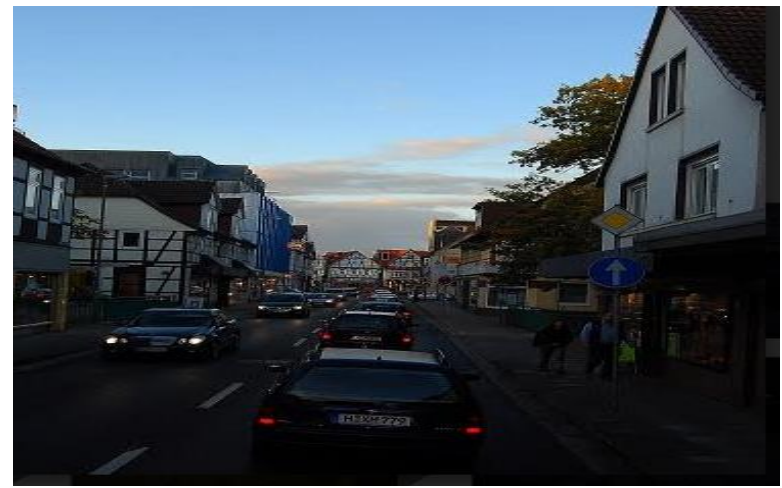

Figure.2 Input image

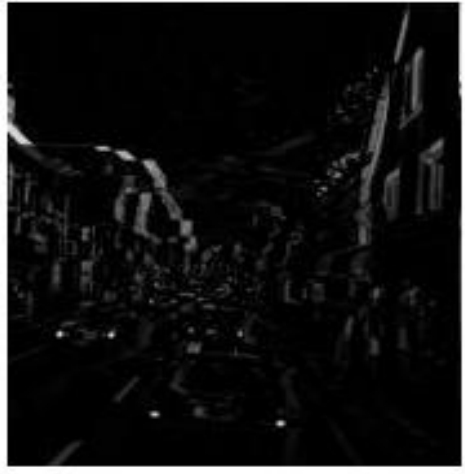

$1^{\text {st }}$ order derivative $(\mathrm{H})$

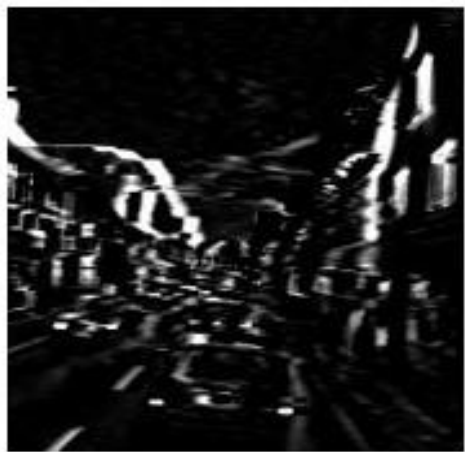

$2^{\text {nd }}$ order derivative $(\mathrm{H})$

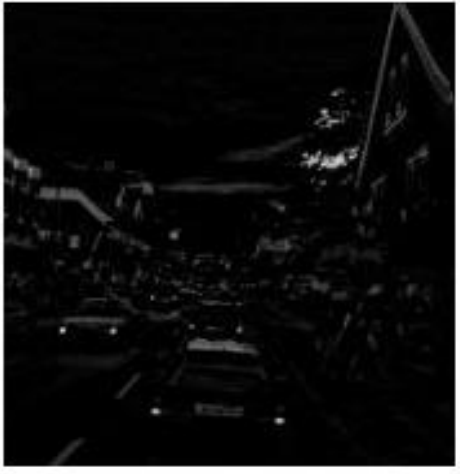

$1^{\text {st }}$ order derivative $(\mathrm{V})$

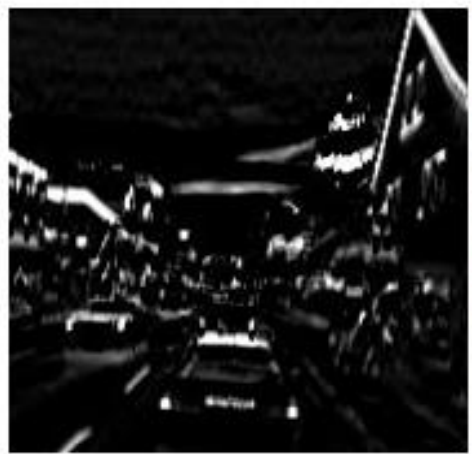

$2^{\text {nd }}$ order derivative $(\mathrm{V})$

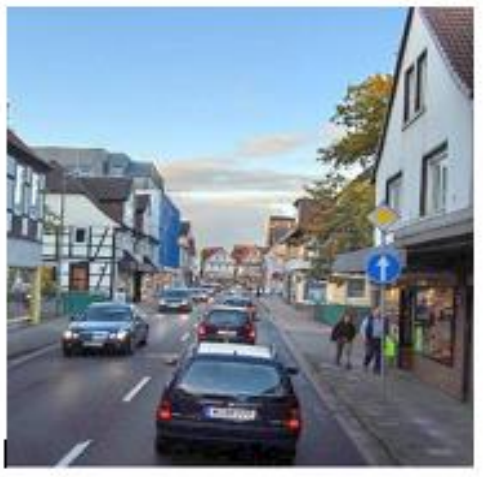

$1^{\text {st }}$ order derivative Output

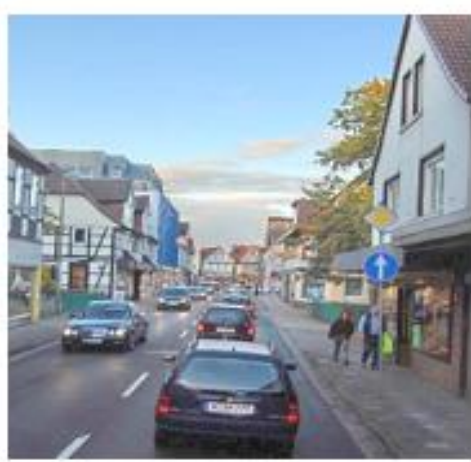

$2^{\text {nd }}$ order derivative Output

Figure.3 Sample image of after mapping function

Fig. 2 indicates the input image of the ICE process and Fig. 3 indicates the first order derivative and second order derivative based mapping function of ICE. In Fig. 2, the first row represents the 1st order derivative performance in horizontal $(\mathrm{H})$ and Vertical (V) way. In Fig. 2, second row represents the 2nd order derivative performance in horizontal $(\mathrm{H})$ and vertical $(\mathrm{V})$ way with final output. The second derivative function helps to identify the position of the pixel edges either dark or light side of an edge. After mapping function, apply the weber's law to adjusting the lighting condition in the image.

\subsection{Lighting Distortion}

In order to avoid the sudden changes in image lighting the logarithmic functions are used such as contrast operators and Weber's law. The Weber's law is mathematically represented as Eq. (12).

$$
C=\frac{L(x)_{\max }-L(x)_{\min }}{L(x)_{\min }}
$$

Whereas, $C$ is contrast of the object, maximum and minimum luminance is noted and indicated as $L(x)_{\max }$ and $L(x)_{\min }$ respectively. The lighting distortion methodology helps to calculate the 


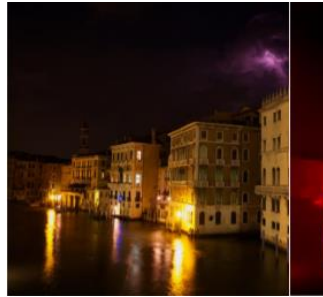

(a)

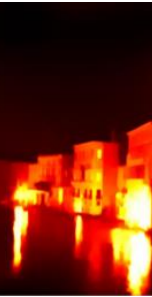

(b)

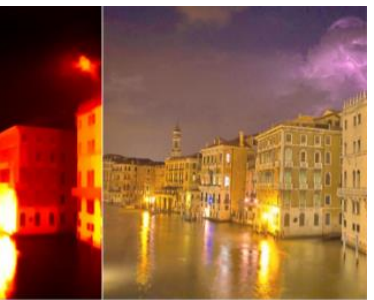

(c)

Figure.4 Sample of image contrast: (a) input image, (b) illumination map, and (c) output mage

average between the smallest and largest regional minima of the image. However, the image background is not detected normally. Hence, image contrast is not able to improve the efficiency with respect to poor lighting image, due to average variations in image background. The sample of proposed methodology based image contrast is shown in Fig. 4.

\section{Experimental result and discussion}

For experimental simulation, MATLAB (version 2017a) was used on PC with $3.2 \mathrm{GHz}$ with i5 processor. In order to estimate the efficiency of proposed algorithm, performance of the proposed method was compared with Low-light Image Enhancement (LIME) method [18] and camera response model with exposure ratio map estimation [19] on the reputed datasets: VV, LIME-data, DICM, and MEF The performance of proposed SODWL method was compared in terms of LOE value and a few state-of-the-art approaches like Multi Scale Retinex with Color Restoration (MSRCR), Naturalness Preserved Enhancement algorithm (NPE), Dehazing based method (Dong), Multideviation Fusion method (MF), Illumination Estimation based method (LIME), and Simultaneous Reflection and Illumination Estimation (SRIE).

\subsection{Evaluation metrics}

\section{- Lightness order error:}

The LOE helps to measure the lightness distortion of enhanced results. LOE is defined in Eq. (13).

$$
L O E=\frac{1}{m} \sum_{x=1}^{m} R D(x)
$$

Where $R D(x)$ is the relative order difference of the lightness between the original image $P$ and its enhanced version $P^{\prime}$ for pixel $x$, which is defined as follows in Eq. (14).

$$
\begin{gathered}
R D(x)= \\
\sum_{y=1}^{m} U(L(x), L(y)) \oplus U\left(L^{\prime}(x), L^{\prime}(y)\right)
\end{gathered}
$$

Where $m$ is the pixel number, $\oplus$ stands for the exclusive OR operator, $L(x)$ and $L^{\prime}(x)$ are the lightness components at location $x$ of the input images and the enhanced images respectively.

\section{- Visual Information Distortion (VID):}

Visual Information Fidelity (VIF) in reverse mode helps to calculate the distortion of visual data. The VID parameter uses the VIF models to calculate the similarity between the original image $C$ and enhanced image $F$. VIF measure is given in Eq. (15).

$$
V I F=\frac{I(C ; F)}{I(C ; E)}
$$

Whereas, $E$ is the image that the human visual system perceives. The mutual information $I(C ; F)$ and $I(C ; E)$ represented as extracted information.

\subsection{Collected dataset}

In experimental analysis, the low-light input image is taken from four different public datasets such as, VV, LIME-data, DICM, and MEF. The dataset descriptions and samples are described below.

VV dataset: This dataset is collected by researcher Dr. Vassilios Vonikakis. He collected images from his daily life activities and those images are used for ICE. Each image in the dataset has two parts that are correctly exposed and underexposed. The sample image is shown in Fig. 5.

LIME-dataset: In this dataset, illumination chromaticity is manually defined with the help of RGB digital counts from color checkers of the base image. The brightest images did not include the overexposed pixels. Moreover, every image set has an average RGB of $3 \times 3$ neighborhood pixels and
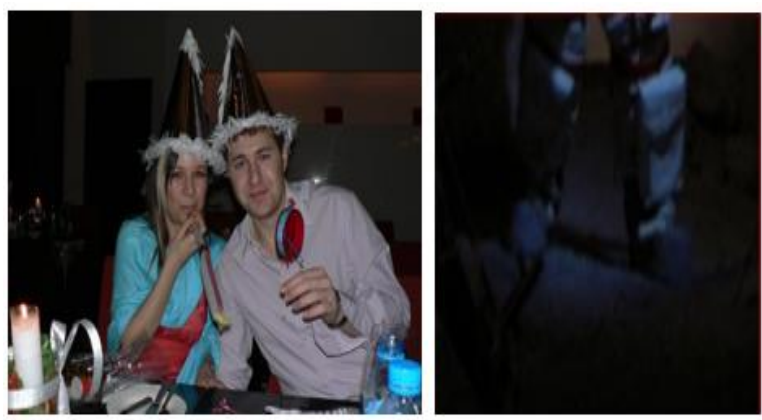

Figure.5 Sample of VV dataset 

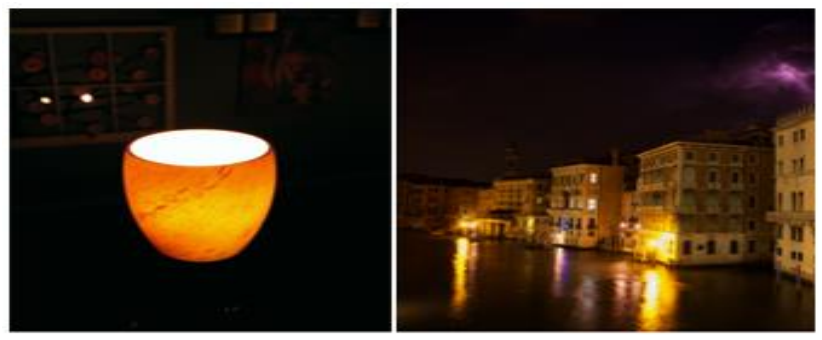

Figure.6 Sample of Lime dataset
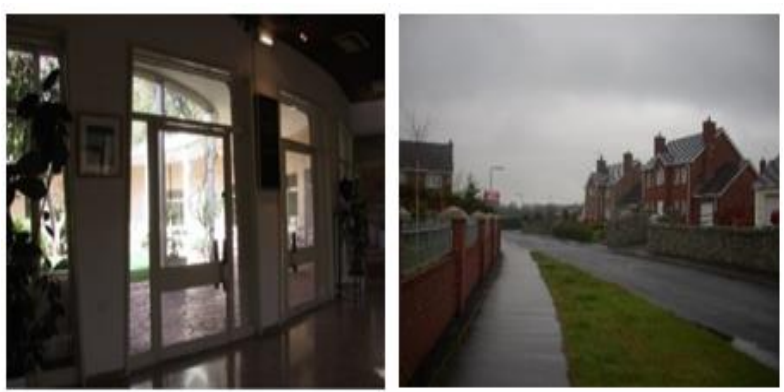

Figure.7 Sample of DICM dataset
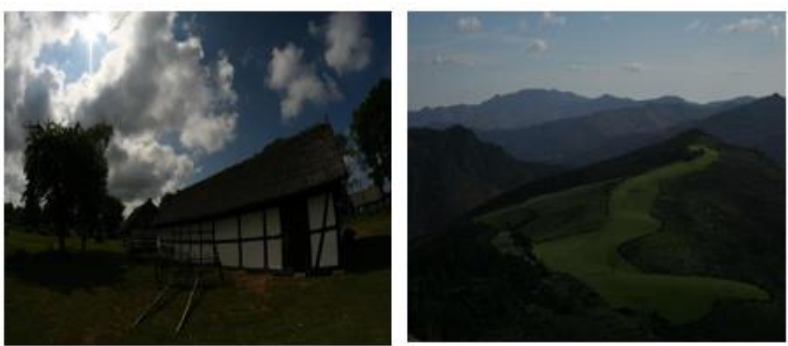

Figure.8 Sample of MEF dataset

center of the white patches are measured. This dataset contains around10 low-light images and all are considered in the experiment. The sample image of LIME dataset is represented in Fig. 6.

DICM dataset: It contains 69 captured images collected from commercial digital cameras and sample images are shown in Fig. 7.
MEF dataset: This dataset contains 17 high-quality image sequences including natural sceneries, indoor and outdoor views and man-made architectures. Each image sequence has several multi-exposure images and select one of the poor-exposed images as input to perform the evaluation. The sample image of MEF dataset is represented in Fig. 8.

\subsection{Quantitative analysis of lightness order error}

In this section, Table 1 contains the LOE value of all datasets on the HDR. From the Table 1, it is observed that the proposed method significantly outperforms the existing methods [18] and [19]. In addition, the visual comparison on different ICE is shown in Fig. 10. According to Fig. 10, it clearly observed that the results obtained by the proposed SODWL method are more visually better and closer to the references than the others. In Table 1, the proposed methodology (SODWL) is analysed for two conditions, when $\gamma=1$ and $\gamma \neq 1$. Compared to the existing methodologies, the proposed method (when $\gamma \neq 1$ ) showed better performance, and achieved LOE of 280 in VV dataset, 471.85 in LIME dataset, 321.24 in DICM dataset, and 293.4 in MEF dataset.

The minimum value of LOE signifies better enhancement of the image in terms of nature of lightness. Due to the heavy load of computing LOE, down-sampling is used to reduce the complexity. For example, the LOE is 0 when no enhancement is performed. Only a few public database includes the ground truth datasets; construction of the ground truth dataset is more complex. The normal version of VIF treats the original image as the reference image and the image generated by the algorithm as the enhanced image. The original input image is the poor visualized with less quality, hence it's difficult to apply in ICE. Therefore, use the VIF in reverse

Table 1. Quantitative performance comparison on the multiple dataset in terms of LOE

\begin{tabular}{|c|c|c|c|c|c|}
\hline \multicolumn{2}{|c|}{ Methods } & VV & LIME data & DICM & MEF \\
\hline \multicolumn{2}{|c|}{ MSRCR [18] } & 2727.7 & 1835.5 & 1795.3 & 1686.2 \\
\hline \multicolumn{2}{|c|}{ Dong [18] } & 853.35 & 1244 & 1180 & 1065.4 \\
\hline \multicolumn{2}{|c|}{ NPE [18] } & 820.93 & 1471.3 & 662.29 & 1158.2 \\
\hline \multicolumn{2}{|c|}{ LIME [18] } & 1274.7 & 1323.8 & 1260.8 & 1079.4 \\
\hline \multicolumn{2}{|c|}{ MF [18] } & 470.93 & 629.82 & 667.45 & 525.95 \\
\hline \multicolumn{2}{|c|}{ SRIE [18] } & 551.39 & 823.61 & 623.3 & 754.2 \\
\hline \multicolumn{2}{|c|}{ Bio-Inspired Framework [18] } & 287.22 & 478.57 & 351.8 & 325.8 \\
\hline \multicolumn{2}{|c|}{$\begin{array}{c}\text { Camera response model } \\
\text { with exposure ratio map } \\
\text { estimation [19] }\end{array}$} & 380 & 437 & - & 295 \\
\hline \multirow{2}{*}{$\begin{array}{l}\text { Proposed } \\
\text { SODWL }\end{array}$} & $\gamma=1$ & 285.82 & 473.30 & 325.6 & 299.54 \\
\hline & $\gamma \neq 1$ & 280 & 471.85 & 321.24 & 293.4 \\
\hline
\end{tabular}


Table 2. Quantitative performance comparison on the multiple dataset in terms of VIF

\begin{tabular}{|c|c|c|c|c|c|}
\hline \multicolumn{2}{|c|}{ Methods } & VV & LIME data & DICM & MEF \\
\hline \multicolumn{2}{|c|}{ MSRCR [18] } & 0.421 & 0.240 & 0.449 & 0.279 \\
\hline \multicolumn{2}{|c|}{ Dong [18] } & 0.504 & 0.325 & 0.526 & 0.353 \\
\hline \multicolumn{2}{|c|}{ NPE [18] } & 0.690 & 0.508 & 0.724 & 0.523 \\
\hline \multicolumn{2}{|c|}{ LIME [18] } & 0.349 & 0.205 & 0.414 & 0.227 \\
\hline \multicolumn{2}{|c|}{ MF [18] } & 0.724 & 0.447 & 0.707 & 0.512 \\
\hline \multicolumn{2}{|c|}{ SRIE [18] } & 0.659 & 0.521 & 0.678 & 0.553 \\
\hline \multicolumn{2}{|c|}{ Bio-Inspired Framework [18] } & 0.760 & 0.742 & 0.745 & 0.600 \\
\hline \multirow{2}{*}{$\begin{array}{l}\text { Proposed } \\
\text { SODWL }\end{array}$} & $\gamma=1$ & 0.832 & 0.501 & 0.904 & 0.629 \\
\hline & $\gamma \neq 1$ & 0.852 & 0.5054 & 0.912 & 0.648 \\
\hline
\end{tabular}
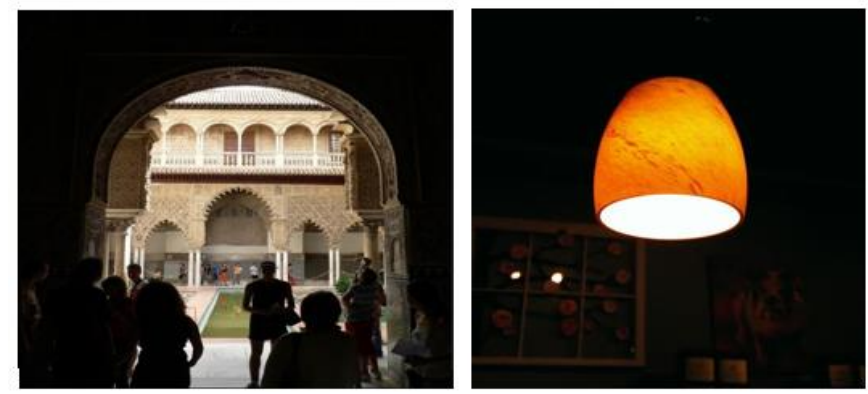

Figure. 9 Visual Comparison of VV data sample and LIME data sample

mode by specifying the enhanced version of the image as the reference and the original image as the degraded image.

VIF provides a consistently high value of correlation between subjective MOS (Mean Opinion Score) and its scores represents the information about the visual quality of the images. The existing non-liner ICE technique and proposed SODWL method performance is tabulated in Table 2 . Compared to the existing methods, the proposed method (when $\gamma \neq 1$ ) showed better performance, and achieved VIF of 0.852 in VV dataset, 0.5054 in LIME dataset, 0.912 in DICM dataset, and 0.648in MEF dataset.

According to the Tables 1 and 2, the existing methods achieved better result compared to proposed method in LIME dataset. The results of LIMEs image data suffer from over enhancement and the color distortions that are relatively high. The proposed framework reasonably enhances the input images and obtain the enhanced results with low VIF value. Although the results of LIME datasets are visually good, and effectively removes the lightness distortions. In other remaining datasets, the proposed methodology evaluates the both non-linear and linear properties of input data and preserves the quantitative relationships between the high and low level pixel values in order to achieve better result compared to the existing methods. The visual sample of VV data and LIME data is shown in Fig. 9.

\section{Conclusion}

In digital image processing, HDR images are the active research area and significantly used in the rapidly growing fields such as film industry, computer graphics, etc. In past decades, ICE approaches are highly used for image enhancement, but the improvements are not defined well. In this paper, an efficient ICE method is proposed namely Second order derivative with Weber's law. The SODWL method helps to improve the visual quality and also to achieve sub-standard quality of the images. The experimental analysis demonstrated that the SODWL method performance measured in efficient evaluation metric namely LOE and performance were compared with the traditional method such as MSRCR, NPE, and LIME. The SODWL method achieved 285.8, 473.3, 325.6, and 299.5 of LOE with respect to VV, LIME, DICM, and MEF. In the future, the research work can be extended by improving the ICE using an efficient optimization technique. 

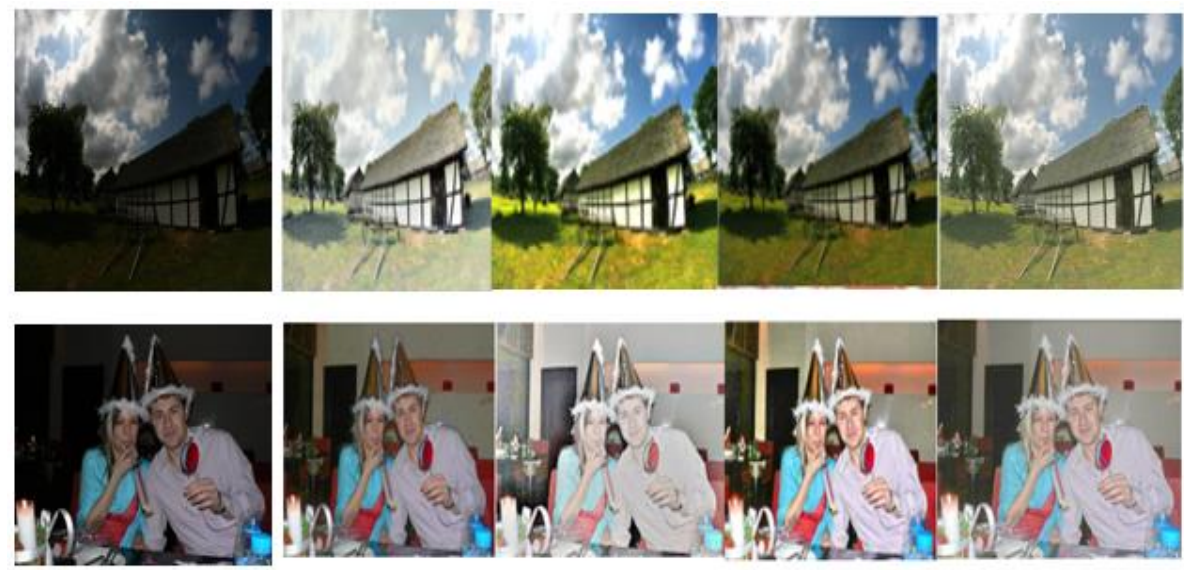

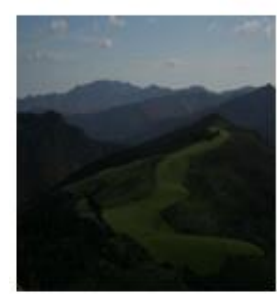

Input

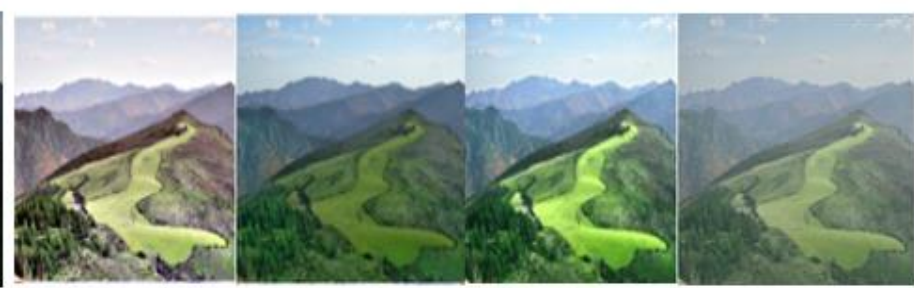

MSRCR

NPE

LIME

Proposed

Figure.10 Visual compression of different methods

\section{References}

[1] M. Sundaram, K. Ramar, N. Arumugam, and G. Prabin, "Histogram modified local contrast enhancement for mammogram images", Applied soft computing, Vol.11, No.8, pp.5809-5816, 2011.

[2] T. Celik and T. Tjahjadi, "Automatic image equalization and contrast enhancement using Gaussian mixture modelling", IEEE Transactions on Image Processing, Vol.21, No.1, pp.145-156, 2012.

[3] S. C. Huang, B. H. Chen, and Y. J. Cheng, "An efficient visibility enhancement algorithm for road scenes captured by intelligent transportation systems", IEEE Transactions on Intelligent Transportation Systems, Vol.15, No.5, pp.2321-2332, 2014.

[4] X. Wang and L. Chen, "An effective histogram modification scheme for image contrast enhancement", Signal Processing: Image Communication, Vol.58, pp.187-198, 2017.

[5] J. Chen, W. Yu, J. Tian, L. Chen, and Z. Zhou, "Image contrast enhancement using an artificial bee colony algorithm", Swarm and Evolutionary Computation, Vol.38, pp.287-294, 2018.

[6] D.S. Prabha and J.S. Kumar, "An efficient image contrast enhancement algorithm using genetic algorithm and fuzzy intensification operator", Wireless
Communications, Vol.93, No.1, pp.223-244, 2017.

[7] Y. Wang and Z. Pan, "Image contrast enhancement using adjacent-blocks-based modification for local histogram equalization", Infrared Physics \& Technology, Vol.86, pp.59-65, 2017.

[8] K. Hasikin and N.A.M. Isa, "Adaptive fuzzy contrast factor enhancement technique for low contrast and nonuniform illumination images", Signal, Image and Video Processing, Vol.8, No.8, pp.1591-1603, 2014.

[9] B.S. Rao, "A fuzzy fusion approach for modified contrast enhancement based image forensics against attacks", Multimedia Tools and Applications, Vol.77, No.5, pp.5241-5261, 2018.

[10] R. Priyadharsini, T.S. Sharmila, and V. Rajendran, "A wavelet transform based contrast enhancement method for underwater acoustic images", Multidimensional Systems and Signal Processing, Vol.29, No.4, pp.1845-1859, 2018.

[11] A.S.A. Ghani, "Image contrast enhancement using an integration of recursive-overlapped contrast limited adaptive histogram specification and dual-image wavelet fusion for the high visibility of deep underwater image", Ocean Engineering, Vol.162, pp. 224238, 2018.

[12] X. Guo, Y. Li, and H. Ling, "LIME: Low-light image enhancement via illumination map 
estimation", IEEE Transactions on Image

Processing, Vol. 26, No.2, pp.982-993, 2017.

[13] X. Liu, G. Cheung, X. Ji, D. Zhao, and W. Gao, "Graph-based joint dequantization and contrast enhancement of poorly lit JPEG images", IEEE Transactions on Image Processing, Vol.28, No.3, pp.1205-1219, 2019.

[14] S. Guo, T. Xiang, and X. Li, "Image quality assessment based on multiscale fuzzy gradient similarity deviation", Soft Computing, Vol.21, No. 5, pp.1145-1155, 2017.

[15] C. Jung, Q. Yang, T. Sun, Q. Fu, and H. Song, "Low light image enhancement with dual-tree complex wavelet transform", Journal of Visual Communication and Image Representation, Vol.42, pp.28-36, 2017.

[16] S. Zhang, T. Wang, J. Dong, and H. Yu, "Underwater image enhancement via extended multi-scale Retinex", Neurocomputing, Vol.245, pp.1-9, 2017.

[17] J. Cai, S. Gu, and L. Zhang, "Learning a deep single image contrast enhancer from multiexposure images", IEEE Transactions on Image Processing, Vol.27, No.4, pp.2049-2062, 2018.

[18] Z. Ying, G. Li, and W. Gao, "A bio-inspired multi-exposure fusion framework for low-light image enhancement", arXiv preprint arXiv:1711.00591, 2017.

[19] Y. Ren, Z. Ying, T.H. Li, and G. Li, "LECARM: Low-Light Image Enhancement using Camera Response Model", IEEE Transactions on Circuits and Systems for Video Technology, Vol.29, No.4, pp.968-981, 2018. 